several species new to science. A particular study will be made of the ecology of the flightless rail, and of the other land bird-a bunting. The populations of the fur and elephant seals will be assessed for the possible future exploitation of the two species.

The invertebrate fauna of Gough Island is almost completely unknown. The isolation of the island, the diversity of its terrain and vegetation, and its comparative freedom from human interference, will make a faunistic survey especially interesting. It is hoped to use the main plant associations as the units in this survey, although a study will be made of the fauna of particular habitats such as streams, caves, beach debris and bird nesting colonies.

The botanist will make a study of the dispersal mechanisms and agents that have brought about the distribution of some of the more interesting plants ; he will also investigate systems of reproduction in a few plants. Pollen analyses of peat samples and their radiocarbon dating will give some indication of the history of plant-life on the island.

The petrological examination of the lavas of Gough may provide clues as to the nature of the Mid-Atlantic Ridge. A gneissous block of 'continental' origin has been found in the crater of Tristan, and there are rocks with similar 'continental' affinities on St. Helena and Ascension; it is hoped that the rocks of Gough will provide further hints about the nature of the Atlantic floor.

The expedition's collections will be distributed to specialists for identification after the party returns, and individual members will work on their own lines of research. Papers will be published in various scientific journals, and in addition it is hoped to collect and republish all the results in a series of papers under the name of the expedition.

\title{
CEREBRAL ACTIVITY
}

A JOINT meeting of Section I. (Physiology) and Section J (Psychology) of the British Association on September 2 at Bristol took the form of a symposium on cerebral activity. Without being restricted to a particular theoretical issue or to data obtained from a particular discipline, this symposium provided common ground for workers from a number of different fields of inquiry relating to the problems of cerebral mechanisms. The topic chosen by the organizers of the symposium may fairly be said to be one where interdependence of different scientific disciplines is becoming increasingly apparent, and the papers which were read on this occasion were largely focused on this point.

The symposium opened with an account by Dr. C. G. Phillips (Oxford) of the essential established facts of nerve structure and function with which any theory of cerebral activity must come to terms.

Reviewing the progress in neurophysiology since the discovery late in the nineteenth century that the nervous system is built up of independent nerve cells, Dr. Phillips described the function of nerve cells in conducting impulses inwards along the dendrites towards the cell body and outwards along the axon to other nerve cell bodies or their dendrites, or to muscles. Dr. Phillips cited the evidence for a voltage gradient across the plasma membrane of the cell body and its processes and (without committing himself to a particular theory of nourohumoral transmission) outlined the sequence of events whereby signals arriving at the spocialized terminals which aro applied to the dendrites and cell bodios of other nerve cells result in the secretion of chemical transmitter substances which serve either to increase or to decrease this voltage gradient. 'The excitatory or inhibitory effect of these terminals was discussed in relation to the critical potential difference which determines the firing of a self-propagating nervous impulse.

Dr. Phillips emphasized that, in considering the norvo cell as a unit in an information system (for example, as a relay), the complexity of this unit's function must not be overlooked. Although the firing or non-firing of a nervous impulse in accordance with a certain critical voltage difference across the plasma membrane may seem a simple and unitary function, nevertheless this is only the end result of the integration of inhibitory and excitatory effects at hundreds of dendritic contacts. The cell "may be poised near to the critical firing level or it may be 'biassed off' over a range of about twenty millivolts, by preponderating inhibitory action". On one hand, there is variability in the amount of further excitation needed if the cell is to fire at all; on the other, given adequate excitation, the frequency of firing is variable over a wide range.

Dr. Phillips then turned to the question of the organization of particular types of cerebral activity, and mentioned that repetitive activity may cause an enduring facilitation of transmission from cell to cell. In suggesting that such changes could conceivably impose fairly definite pathways on an originally diffuse system of interconnexions, he was careful to point out that particular patterns of activity occurring in a more or less restricted brain region might very well engage different populations of brain cells on different occasions, and that one cannot assume an unvarying anatomical substrate for particular types of cerebral activity. In fact, although certain reflex pathways can be traced and although there is some knowledge of the traffic of impulses in a few wellmarked tracts within the central nervous system, the nervous events which subserve such functions as speech and skilled movemont cannot at present be traced in detail.

Dr. W. Grey Walter (Burden Neurological Institute, Bristol) gave an account of some of his investigations into the function of the spontaneous electrical activity of the brain. He suggested that this activity has an 'administrative' function in cerebral organization and, more specifically, that this activity is concerned with the distribution in the brain of informa. tion derived from the sense organs and that this is best considered as operating on a statistical basis. Ho also maintained that this spontaneous activity is related to temperament and social behaviour.

After stressing the inadequacy of the tools which are available for the study of cerebral mechanisms, Dr. Grey Walter pointed out that useful information can nevertheless be gleaned provided that the information extracted by one's recording instruments bears a recognizable relation to the end result of the function under investigation. Ho suggested, for example, that the conventional voltage against time record is unsatisfactory, and that frequency analysis yields more meaningful information. He illustrated 
this point by referring to the electrical changes which occur in the brain during word association procedures and during problem solving.

Another technique which Dr. Grey Walter believes is of particular value is the graphic representation of electrical activity from various parts of the brain. A cluster of cathode-ray tubes with revolving time. bases (intensity modulation) can assist in the analysis of the electrical activity of the brain as a whole, since it is then possible to trace functional relationships between different brain areas. Thus Dr. Grey Walter claimed that this technique shows that the alpha rhythm of the brain is not a simultaneous synchronous firing throughout the brain, but that instead the alpha rhythm picked up from different parts of the scalp is not completely in phase. Similarly, a rhythmically flashing light (when the time relations of the apparatus are adjusted to the periodicity of the stimulus) is reflected in a corresponding pattern in parts of the visual display of the apparatus. This pattern may appear in a unitary fashion in a particular part of the cortex (for example, the occipital lobes), may be broken down into components, with these components distributed to different parts of the brain, or may even be conserved (in the temporal region) for a period as long as 10 or 20 sec. after the cessation of the stimulus. The cerebral consequences of visual stimulation as measured with this technique show habituation effects with prolonged stimulation and also dishabituation when subjective monotony is relieved by the introduction of a reward.

Dr. Grey Walter carried interpretation a step further when he claimed that people with similar patterns of electrical activity of the brain tend to have temperamental affinity and are well fitted for social co-operation with each other.

Dr. D. M. Mackay (King's College, London) discussed some of the theoretical problems of cerebral organization. He pointed out that the fault with the kind of analogy which compares the brain to known mechanical or electronic devices is that it is too restricted, and he stressed the necessity for a more basic and general language. This, he urged, could first most properly be employed to describe what the brain does before attention is turned to the problem of how it does it. He suggested that, given adequate definition of what the brain does, one could proceed to consider various possible alternatives as to the mechanism involved and then refer back to the known facts of neurophysiology for guidance as to which of the postulated mechanisms occurs in the brain. The theory of information and control which has arisen from recent developments in communications and automatic control systems is framed in terms which, Dr. Mackay suggested, are of sufficient generality to meet the requirements he had referred to. This basic terminology, he maintained, could be applied to both physiological and psychological data, possessed a reasonable chance of surviving changes in engineering fashion, and lent itself particularly woll to a theory concerned with units with a controllable probability of passing on signals (as described by Dr. Phillips).

Dr. Mackay raised two important issues which would necessarily have to govern any theoretical model of cerebral organization : first. such a model would need to be self-organizing; secondly, it would need to be able to take advantage of the redundancy of information which is implicit in a stable environment. It would thus be necessary to work out the general principles upon which such a system as the brain would have to function if it is to take advantage of the statistical regularities of the world with which it has to deal. Similarly, Dr. Mackay argued, the question of the time which such a system would require in order to adjust itself to these regularities is a crucial one. Thus there is no possibility of a model of restricted size which could make the necessary adjustments by simple trial, error and success in relation to each alternative with which it is confronted. A principle which would be more consistent with the observed facts of behaviour, Dr. Mackay contended, is one where the system starts with "the degrees of freedom coalesced", so that the process of self-development becomes one where the probability of any particular event occurring would be reduced rather than increased during the period of organization. Dr. Mackay's suggestion is thus nearer to Coghill's theory of differentiation through maturation than to learning in terms of conditioning or simple trial-and-error learning. Finally, Dr. Mackay sug. gested that such a model might find its first application and its first test in relation to the effects of brain injuries, since the types of breakdown which might occur with any particular model should be predictable.

Mr. M. F. Piercy (Bristol) discussed behavioural studies of cerebral dysfunction. He pointed out that the behaviour of a normal organism in a normal environment gives very little clue as to the changed behaviour which may result from physical interference with cerebral function. Similarly, this behaviour would not be predictable on the basis of present neuro-physiological knowledge. This situation, he suggested, provides a starting-point for studies which may eventually modify both psychological and physiological theories.

As an example of studies taking their cue from clinical neurology, he cited work on the incidence of aphasia in relation to cerebral dominance. Although, in right-handed patients, aphasia occurs almost exclusively in association with lesions of the left hemisphere, nevertheless left-handed patients may sustain an aphasia as a result of a lesion of either hemisphere. There is however, he maintained, evidence to suggest that aphasia is often less severe and more transient in left-handed people. In distinction to this evidence, Mr. Piercy referred to a study of paroxysmal dysphasia (with which he had been concerned). This showed that paroxysmal aphasia occurring in association with a short-lived unilateral cerebral disturbance was more frequent in left-handed than in right-handed patients, irrespective of the side of the focal cerebral disturbance. It may be, he argued, that if a function is more diffusely organized with respect to cerebral anatomy (for example, language in left-handers), not only is restitution of function noore easily achieved when the disturbance is ehronic, but also that function is more liable to disruption in the face of acute interference. Conversely, in the case of a function being organized within a more restricted cerebral area (for example, language in right-handers), Mr. Piercy suggested that the function might be more resistant to acute interference (such as paroxysmal) but less capable of recovery when breakdown occurs in association with a chronic lesion. This, he said, was a tentative theoretical position which should be amenable to experimental verification or disproof.

Mr. Piercy then directed attention to studies which produced anomalous behaviour without direct interference with cerebral function. Thus, if a person's 
voice is recorded and played back to him through earphones with a delay of about half a second, gross disturbances of speech are liable to occur. These disturbances are not unlike those occurring in some forms of dysphasia. Similarly, a recent study carried out in Canada has shown that if sensory stimulation is reduced to a minimum for a period of hours or days, a human subject may experience hallucinations and feelings of unreality which closely resemble those occurring under the influence of hallucinogenic drugs. Similar symptomatology from radically different experimental procedures may or may not mean that a common cerebral mechanism is disturbed; but such converging lines of evidence should, he urged, at least provide strong stimulus for further research.
Malcolm Piercy

\section{PROGRESS OF CANCER RESEARCH}

$\mathrm{T}$ HE annual report of the British Empire Cancer Campaign has now expanded in its 1955 edition* to more than 500 pages; consequently, this short review can only deal with a very limited number of the researches financed and organized by the Campaign. The investigations to be described can be divided into three main groupings, namely, those concerned with carcinogenesis and other studies of that kind, elinico-pathology, and radiology.

In the editorial summary to the report there is a statement which says that "steady progress continues in our understanding of the manner in which cancer-producing substances act". Unfortunately, this happy thought verges on the over-optimistic.

Work on the fashionable cancer problem, the lung cancer-cigarette smoking relationship, continues at a number of centres. One of the main difficulties, from the experimentalist's point of view, is to find some tissue which has the same sensitivity as human lung and upon which the carcinogens present in cigarette smoke could be tested. Sir Ernest Kennaway and co-workers have found $3: 4$. benzpyrene, anthracene, pyrene and acenaphthylene in the condensate from tobacco smoke, and the same compounds have also been detected in smoke from the paper in which the cigarette tobacco is rolled. Sir Ernest has attacked the difficulty of test material by using tissue-culture technique :

"Several workers have demonstrated the carcinogenic power of tobacco condensates upon the skin of the mouse, but neither the species nor the tissue concerned in these experiments is of practical importance. Human tissue ean be used thus only in tissue culture. Dr. Honor B. Fell, of the Strangeways Research Laboratory, Cambridge, has kindly allowed Dr. Ilse Lasnitzki to investigate this matter. Lung tissue obtained from an early human foetus, and therefore sterile, was found to grow well in culture without replacement of epithelium by fibroblasts.

"Such cultures are being exposed to $3: 4$-benzpyrene, and to various fractions of tobacco condensates, in order to learn whether the nuclear changes known to be produced by carcinogens in other tissues can be induced in this instance also."

M. H. Salaman and collaborators have reported interesting developments in the study of the two stages of carcinogenesis ('initiation' and 'promotion'). They find that pre-treatment of mouse skin with urethane (initiation), followed by promotion with croton oil, yields skin tumours; however, urethane, although a highly potent carcinogen for mouse lung, does not induce cancer in mouse skin when applied.

* British Empire Cancer Campaign. Thirty-second Annual Report covering the year 1954. Pp. xxx +531. (London: British Empire Cancer Campaign, 1955.) alone. It has now been found that for the initiation stage in mouse skin not only urethane but TEM (trisethyleneimino-3-triazine) or $1: 2$-benzanthracene or $\beta$-propiolactone are also effective. The promoter, too (croton oil), can be replaced by iodoacetic acid or by chloracetophenone.

The search for chemotherapeutic drugs for eancer is undergoing an interesting change; less emphasis is now being placed on the therapeutic results achieved, and more and more on the synthesis and preparation of the drugs and upon the mechanism of their cytotoxic action from the points of view of cytology and physical chemistry.

Dr. Percy Stocks, in a study of the influence of social factors on the incidence of breast cancer in women, states :

"The figures suggest that when marriage is delayed the risk of breast cancer is increased, whatever the number of children borne, except in the group married about 30 with long duration of married life; or, otherwise expressed, that high fertility has a protective effect provided that marriage has not been delayed too long.

"In due course larger numbers will be available to establish this but taking account also of the remarkably low breast cancer rates in Japan where fertility has been high and breast feeding usually very prolonged, sufficient justification has been found for an attempt to seek answers to the following questions :

"(1) Does the mortality excess associated with zero and low fertility indicate simply a greater frequency of breast cancer, or is there also a greater probability of high malignancy when cancer occurs (i.e. higher fatality without higher inception rates) ?

"(2) Is short duration of breast feeding more important than few confinements in producing the excess ?"

There are no surprises in the field of morbid anatomy this year. It is good to learn that Bloom's series of cancers of the breast approaches the thousand mark, and that its full statistical analysis is being undertaken. The extraordinary statistical difficulties which bristle about every attempt to assess the effects of different forms of treatment of this disease, and the effect of varying histology, can only be solved by study of really large and uniformly recorded series such as we can hope for in this case. D. H. Patey and A. C. Thackray provide a much-needed study of the whole gland resected in cases of parotid tumour: in none of their cases (save their only adenolymphoma) was there any evidence whatever of multicentric origin-a most important point to be settled in regard to treatment. It is, however, disappointing to find they have used only ten cases.

The review of the tumours of childhood from the Hospital for Sick Children at Great Ormond Street, 